\title{
Gravitational cells and gravitational strings as a necessary part of the gravitational field. Obtaining new physical formulas and indicators (the formula for the gravitational constant, the formula for the mass of the hydrogen atom, etc.)
}

Andrey Chernov ( $\square$ and8591@gmail.com ) none Company/Institution

\section{Article}

Keywords: Black hole, gravitational cell, gravitational string, gravitational quantum, gravitational constant formula, the proportion between the mass of the electron and the proton, electron mass formula, hydrogen atom mass formula, elementary charge, Schwarzschild radius formula, gravitational cell mass, the gravitational field, gravitational constant.

Posted Date: November 1st, 2021

DOI: https://doi.org/10.21203/rs.3.rs-622706/v16

License: (1) This work is licensed under a Creative Commons Attribution 4.0 International License. Read Full License 
Gravitational cells and gravitational strings as a necessary part of the gravitational field. Obtaining new physical formulas and indicators (the formula for the gravitational constant, the formula for the mass of the hydrogen atom, etc.).

\section{Author Andrey Chernov}

Address: Andrey Chernov, 16 Polenova str, apt.7, Irkutsk, 664007, Russia. cell phone: +7 9645407 298. E mail: and8591@gmail.com

\section{Content}

1. Abstract -2 pg.

2. Introduction $-2-3 \mathrm{pg}$.

3. Methods $-3-15 \mathrm{pg}$.

4. Results -15 pg.

5. Conclusions $-16 \mathrm{pg}$.

6. Declarations $-17 \mathrm{pg}$. 


\begin{abstract}
.
In this work, for the first time, it was possible to build into the gravitational field such concepts as gravitational cells and gravitational strings. This made it possible to obtain such scientific results as the formula for the gravitational constant, the formula for the proportion between the mass-energy of the electron and the proton, the formula for the hydrogen atom, the formula for the gravitational quantum, etc. These formulas were fully confirmed by experimental data. In this study, Planck's formula was successfully embedded in the gravitational field, resulting in significant scientific results. In this work, the concept of a gravitational quantum is introduced and its value is determined. Also, a new physical constant was obtained - the mass of the gravitational cell of a black hole.
\end{abstract}

Keywords. Black hole, gravitational cell, gravitational string, gravitational quantum, gravitational constant formula, the proportion between the mass of the electron and the proton, electron mass formula, hydrogen atom mass formula, elementary charge, Schwarzschild radius formula, gravitational cell mass, the gravitational field, gravitational constant.

\title{
Introduction.
}

This study envisages embedding into the concept of the gravitational field, such physical concepts as gravitational cells and gravitational ones. This will make it possible to move from general concepts of the gravitational interaction of bodies 
in space to a more detailed understanding of this physical process and to obtain confirmed scientific results.

\section{Methods.}

The gravitational field of any body cannot be considered separately without taking into account the interaction of this body with another body in space. In this case, the magnitude of the gravitational field depends not only on the amount of matter (mass), but also on the structure of the interacting bodies. This structure includes gravitational cells. These cells have a charge equal to two elementary charges $\mathbf{2} \boldsymbol{q}$ and a mass $\boldsymbol{m}$. Each gravitational cell $\mathrm{m}$ forms around itself in space a set of point gravitational fields $\boldsymbol{g}_{\boldsymbol{n}}$, the intensity of which depends on the distance $\boldsymbol{r}_{\boldsymbol{n}}$ to this point, that is, $\boldsymbol{g}_{\boldsymbol{n}}=\frac{2 \boldsymbol{q}}{\boldsymbol{r}_{\boldsymbol{n}}^{2}}$. These numerous point fields are up to a certain point latent potential fields and can manifest themselves only upon contact with the gravitational cells of other bodies. Therefore, when another body of mass $\boldsymbol{M}_{\mathbf{1}}$ (also consisting of a plurality of gravitational cells with a charge of $\mathbf{2 q}$ ) hits this area of space, a real gravitational field now appears at the point of contact of two cells: $\boldsymbol{g}=\frac{\boldsymbol{k} 2 \boldsymbol{q} \cdot \boldsymbol{k} 2 \boldsymbol{q}}{\boldsymbol{r}^{2}}=\frac{\boldsymbol{k}^{2} 4 \boldsymbol{q}^{2}}{\boldsymbol{r}^{2}}$. As a result, the field $\boldsymbol{g}$ imparts the acceleration $\boldsymbol{g}=$ $\frac{\boldsymbol{k}^{2} \mathbf{4} \boldsymbol{q}^{2}}{\boldsymbol{r}^{2}}$ to the gravitational cell of the body $\boldsymbol{M}_{1}$. Due to the fact that the body $\boldsymbol{M}_{1}$ consists of a huge number of such cells with a charge of $\mathbf{2} \boldsymbol{q}$, and the dimensions of the body are much less than the distance $\boldsymbol{r}$ between $\boldsymbol{M}$ and $\boldsymbol{M}_{\mathbf{1}}$, then the whole body $\boldsymbol{M}_{\mathbf{1}}$ will experience an acceleration in this area of space equal to the magnitude of the acceleration of one gravitational cell, that is, $g=\frac{\boldsymbol{k}^{\mathbf{2}} \mathbf{4 q ^ { 2 }}}{\boldsymbol{r}^{\mathbf{2}}}$. (This 
situation can be compared with the same accelerated motion of many absolutely identical charged particles in an electric field of a large electric charge).

Now let's move on to the basic formulas. A body of mass $\boldsymbol{M}$ consists of a huge number of $\boldsymbol{n}$ gravitational cells of mass $\boldsymbol{m}$, where $\boldsymbol{n}=\frac{\boldsymbol{M}}{\boldsymbol{m}}$. These cells together form a common gravitational field $\boldsymbol{E}=\boldsymbol{g} \cdot \boldsymbol{n}$. As a result of all of the above, the formula for the gravitational field of a body with mass $\boldsymbol{M}$ when it interacts with another mass $\boldsymbol{M}_{\mathbf{1}}$ looks like this:

$$
E=g \cdot n=\frac{k^{2} 4 q^{2}}{r^{2}} \frac{M}{m}
$$

where $\boldsymbol{E}$ is the gravitational field of the body $\boldsymbol{M}, \mathrm{m} / \mathrm{s}^{2}$.

$\boldsymbol{g}$ is the value of the field of one gravitational cell of the body $\boldsymbol{M}, \mathrm{m} / \mathrm{s}^{2}$.

$\boldsymbol{m}$ is the mass of the gravitational cell of the body $\boldsymbol{M}, \mathrm{kg}$.

$\boldsymbol{n}$ is the number of gravity cells in the mass $\boldsymbol{M}$.

$\boldsymbol{q}$ is the value of elementary charges, where $\boldsymbol{q}=\mathbf{1 , 6 0 2 1 7 7 3 3} \cdot \mathbf{1 0}^{-\mathbf{1 9}} \mathrm{Cl}$.

$\boldsymbol{k}$ - coefficient of proportionality of charges, $\frac{\mathrm{m}^{3 / 2}}{\mathrm{C} \mathrm{s}}$

The field interaction of two gravitational cells in space can be considered as an extended power string. The energy of such a gravitational string is $\boldsymbol{e}=$ $\boldsymbol{k}^{\mathbf{2}} \mathbf{4} \boldsymbol{q}^{\mathbf{2}}$. Hence the formula (1-1) will take the following form:

$$
E=K \frac{e}{r^{2}} \frac{M}{m} \quad \text { or } E=\frac{e}{r^{2}} \frac{M}{m}
$$

where $\boldsymbol{e}$ is the energy of the gravitational string between the cells, J. $\boldsymbol{K}$ - coefficient for converting units of measurement, where $\boldsymbol{K}=\mathbf{1} \mathrm{m} / \mathrm{kg}$. 
In the future, in order to avoid confusion with other proportionality coefficients $\boldsymbol{k}$, the conversion factor of units of measurement $\boldsymbol{K}=1$ will not be reflected in the formulas. Note also that if in the formula (1-2) the expression $\frac{\boldsymbol{e}}{r^{2}}$ is denoted as $\boldsymbol{G}$, then we get the familiar formula of the gravitational field: $\boldsymbol{E}=\boldsymbol{G} \frac{\boldsymbol{M}}{\boldsymbol{r}^{2}}$.

To clearly understand the physics of the process, it is necessary first to consider the case of the gravitational interaction of two superdense masses, called black holes. So, we have two superdense masses $\boldsymbol{M}$ and $\boldsymbol{M}_{\mathbf{1}}$, located at a distance r from each other. These two masses are a homogeneous substance, consisting of many superdense cells with mass $\boldsymbol{m}_{\mathbf{0}}$ and a charge equal to the value of two elementary charges - $\mathbf{2} \boldsymbol{q}$. Such gravitational cells were formed after an extremely dense compression of matter, as a result of which molecules and atoms turned into identical gravitational cells, consisting of two opposite charges. (The mass of such a cell should be less than the total mass of a free proton and a free electron by $\Delta \boldsymbol{m}$ due to the release of energy during the compression of matter, where $\Delta \boldsymbol{m}=$ $\left.\boldsymbol{E} / \mathrm{c}^{2}\right)$. The formula of the gravitational field $\boldsymbol{E}$ of a superdense body with mass $\boldsymbol{M}_{\mathbf{0}}$ in its interaction with another superdense body $\boldsymbol{M}_{\mathbf{0 1}}$ looks like formula (1-2):

$$
E=\frac{e_{0}}{r^{2}} \frac{M_{0}}{m_{0}}
$$

$\boldsymbol{e}_{\mathbf{0}}$ is the energy of the gravitational string between two cells, J.

$\boldsymbol{m}_{\mathbf{0}}$ is the mass of the gravitational cell, $\mathrm{kg}$.

In expanded form, the formula (1-3) looks like this:

$$
E=\frac{k_{0}^{2} 4 q^{2}}{r^{2}} \frac{M_{0}}{m_{0}}
$$


$\boldsymbol{k}_{\mathbf{0}}$ is the proportionality coefficient of the charges in the cell, where $\boldsymbol{k}_{\mathbf{0}}=\mathbf{1}$.

(hence $e_{0}=4 q^{2}=1,026789 \cdot 10^{-37} \mathrm{~J}$ ).

$\boldsymbol{q}$ - elementary charge, $\mathbf{1 , 6 0 2 1 7 7 3 3} \cdot \mathbf{1 0}^{-19} \mathrm{Cl}$.

Formula (1-4) shows that in a superdense state of matter, the proportionality coefficient of charges is $\boldsymbol{k}_{\mathbf{0}}=\mathbf{1}$ (for comparison: when electric charges interact in a vacuum, $\boldsymbol{k}=\mathbf{9} \cdot \mathbf{1 0}^{\mathbf{9}}$ ). The reason for such a large discrepancy lies in the fact that the substance of the black hole is so strongly compressed that almost all lines of force of elementary charges are closed inside gravitational cells. And only an extremely small part of the lines of force goes out, creating a gravitational field in the outer space. As a result of this circumstance, the coefficient of proportionality of elementary charges outside the cell decreases to its minimum threshold, that is, exactly to 1 . In this case, the main field, with the coefficient of proportionality $\boldsymbol{k}=$ $\mathbf{9} \cdot \mathbf{1 0}^{9}$, remains closed between the elementary charges inside the gravitational cell and therefore does not manifest itself in any way.

In the formula (1-3), the expression $\frac{\boldsymbol{e}_{\mathbf{0}}}{\boldsymbol{m}_{\mathbf{0}}}$ shows the value of the gravitational constant, where $G_{0}=\frac{e_{0}}{m_{0}}$.

If we accept the condition that the gravitational constant in the black hole region $G_{0}=G=6,6743 \cdot 10^{-11}$, then we get:

$$
m_{0}=\frac{e_{0}}{G_{0}}=\frac{1,026789 \cdot 10^{-37}}{6,6743 \cdot 10^{-11}}=1,538422 \cdot 10^{-27} \mathrm{~kg}
$$

But such a result $\boldsymbol{m}_{\mathbf{0}}$ cannot be considered final, because the gravitational constant $\boldsymbol{G}_{\mathbf{0}}$ under extreme conditions of a black hole may have a different value. 
Therefore, for the sake of purity of the study, the obtained value $\boldsymbol{m}_{\mathbf{0}}=$

$\mathbf{1 , 5 3 8 4 2 2} \cdot \mathbf{1 0}^{-\mathbf{2 7}} \mathrm{kg}$ should be checked through another formula associated with the concept of "black hole". Such a test formula is the Schwarzschild radius formula.

$$
R=\frac{2 G_{0}}{c^{2}} \cdot M
$$

where $\boldsymbol{R}$ is the gravitational radius of a black hole, $\mathrm{m}, \boldsymbol{G}_{\mathbf{0}}$ is the gravitational constant in the field of a black hole, $\boldsymbol{M}$ is the mass of a black hole, $\mathrm{kg}$, $\boldsymbol{c}$ is the speed of light, $\mathrm{m} / \mathrm{s}$.

In this formula, the expression $\frac{2 G_{0}}{c^{2}}$ is of particular interest. This expression is equal to $\frac{\boldsymbol{R}}{\boldsymbol{M}}$, measured in "m / $\mathbf{k g}$ " and is a specific indicator of "length" and "mass". When multiplying $\frac{2 G_{0}}{c^{2}}$ by the mass of the body $\boldsymbol{M}$, the gravitational radius of the black hole is determined. But in the one-dimensional space of a black hole, such a physical quantity as length does not exist, therefore the $\frac{\boldsymbol{R}}{\boldsymbol{M}}$ index in "m / $\mathbf{k g}$ " should be perceived as the minimum structural unit of the black hole substance, that is, the mass of the gravitational cell $\boldsymbol{m}_{\mathbf{0}}$. It follows that $\boldsymbol{m}_{\mathbf{0}}=\frac{\mathbf{2} \boldsymbol{G}_{\mathbf{0}}}{\boldsymbol{c}^{\mathbf{2}}}$. Taking into account the fact that according to f. (1-5) $\boldsymbol{m}_{\mathbf{0}}=\frac{\boldsymbol{e}_{\mathbf{0}}}{\boldsymbol{G}_{\mathbf{0}}}$, we get the following equation: $\frac{2 G_{0}}{c^{2}}=\frac{e_{0}}{G_{0}}$

Let's solve this equation and get:

$$
\begin{aligned}
& G_{0}=\sqrt{2} q c=6,7927 \cdot 10^{-11} \\
& m_{0}=\frac{\sqrt{8 q}}{c}=1,511593 \cdot 10^{-27} \mathrm{~kg}
\end{aligned}
$$


As you can see, the mass of the gravitational cell of the black hole is $\boldsymbol{m}_{\mathbf{0}}=$ $\mathbf{1 , 5 1 1 5 9 3} \cdot \mathbf{1 0}^{-\mathbf{2 7}} \mathrm{kg}$, and not , $\mathbf{5 3 8 4 2 2} \cdot \mathbf{1 0}^{-\mathbf{2 7}} \mathrm{kg}$, as calculated above. But at the same time, these very close results, which were obtained in different ways, indicate the correctness of the hypothesis of gravitational cells. When choosing between two values of $\boldsymbol{m}_{\mathbf{0}}$, it will be more correct to dwell on the value obtained using the Schwarzschild formula, that is, $\boldsymbol{m}_{\mathbf{0}}=\mathbf{1}, \mathbf{5 1 1 5 9 3} \cdot \mathbf{1 0}^{-\mathbf{2 7}} \mathrm{kg}$. (This will be confirmed by calculations in this article when determining the mass of a hydrogen atom).

The discrepancy between $\boldsymbol{G}_{\mathbf{0}}=\mathbf{6}, \mathbf{7 9 2 7} \cdot \mathbf{1 0}^{-\mathbf{1 1}}$ and $\boldsymbol{G}=\mathbf{6 , 6 7 4 3} \cdot \mathbf{1 0}^{-11}$ is only $\mathbf{1 , 7 \%}$. This slight difference is due to structural changes in superdense gravity cells.

Now let us consider the gravitational interaction of an "ordinary" body of mass $\boldsymbol{M}$ with another "ordinary" mass $\boldsymbol{M}_{\mathbf{1}}$. The formula of the gravitational field $\boldsymbol{E}$ of the body $\boldsymbol{M}$ according to the basic formula (1-2) looks like this:

$$
E=\frac{e}{r^{2}} \frac{M}{m}
$$

$\boldsymbol{e}$ is the energy of the gravitational string between the cells, where $\boldsymbol{e}=$ $1,108293 \cdot 10^{-37} \mathrm{~J}$

$\boldsymbol{m}$ is the mass of the gravitational cell, where $\boldsymbol{m}=\mathbf{1 , 6 6 0 5 3 9} \cdot \mathbf{1 0}^{-27} \mathrm{~kg}$.

In expanded form, this formula looks like this:

$$
E=\frac{k^{2} 4 q^{2}}{r^{2}} \frac{M}{m}
$$


where $\boldsymbol{k}$ is the proportionality coefficient of the charges of the gravitational cell, where $\boldsymbol{k}=1,038931 \frac{\mathrm{m}^{3 / 2}}{\mathrm{C} \mathrm{s}}, \boldsymbol{k}^{2}=1,079378 \frac{\mathrm{m}^{3}}{\mathrm{C}^{2} \mathrm{~s}^{2}}$

From here we obtain the classical formula of the gravitational field: $\boldsymbol{E}=\boldsymbol{G}$ $\frac{M}{r^{2}}$, where $G=\frac{e}{m}=6,6743 \cdot 10^{-11}$.

Let us now explain the quantities $\boldsymbol{k}$ and $\boldsymbol{m}$. To do this, imagine that ordinary matter was formed from the superdense matter of a black hole. In this case, each superdense gravitational cell, due to the influx of energy $\boldsymbol{E}$, will increase its mass $\boldsymbol{m}_{\mathbf{0}}$ to mass $\boldsymbol{m}$ by the amount $\Delta \boldsymbol{m}$ (where $\Delta \boldsymbol{m}=\boldsymbol{E} / \mathrm{c}^{2}$ ). As a result, a plasma is formed from a superdense substance, from which gaseous, liquid and solid substances can then be formed. All four states of matter are neutral, that is, they have a total electric charge equal to zero. As a result of this circumstance, any "ordinary" substance can be represented as a huge set of gravitational cells. All four states of matter are neutral, that is, they have a total electric charge equal to zero. As a result of this circumstance, any "ordinary" substance can be represented as a huge set of gravitational cells. These cells consist of a proton and an electron with a total charge of $\mathbf{2} \boldsymbol{q}$, as well as of neutrons, which are also a pair of a proton and an electron with a total charge of $\mathbf{2} \boldsymbol{q}$. Thus, the mass of the gravitational cell $\boldsymbol{m}$ of any substance (plasma, gas, liquid or solid) with a high degree of accuracy will be equal to $\mathbf{1} \mathrm{Da}$ or $\boldsymbol{m}=\mathbf{1 , 6 6 0 5 3 9} \cdot \mathbf{1 0}^{-\mathbf{2 7}} \mathrm{kg}$.

Under the conditions of the standard density of the substance, $\boldsymbol{k}=$ $\mathbf{1}, \mathbf{0 3 8 9 3 1}$, that is, $\boldsymbol{k}>\boldsymbol{k}_{\mathbf{0}}=\mathbf{1}$. This very small difference between $\boldsymbol{k}$ and $\boldsymbol{k}_{\mathbf{0}}$ can be explained by the fact that, in contrast to the gravitational cell of a black hole, 
where elementary charges are absolutely tightly adjacent to each other, in an ordinary cell there is some ultramicroscopic distance between two elementary charges. As a result of this circumstance, a little more lines of force come out of an ordinary cell, as a result of which $\boldsymbol{k}>\boldsymbol{k}_{\mathbf{0}}$.

The gravitational interaction of an "ordinary" body of mass $\boldsymbol{M}$ and a superdense body of mass $\boldsymbol{M}_{\mathbf{0}}$ is determined by the total value $\boldsymbol{e}_{\mathbf{1}}=\boldsymbol{k}^{\mathbf{2}} \mathbf{4} \boldsymbol{q}^{\mathbf{2}}=$ $\mathbf{1}, \mathbf{1 0 8 2 9 3} \cdot \mathbf{1 0}^{-37} \mathrm{~J}$ and different masses of gravitational cells $\boldsymbol{m}=$ $1,660539 \cdot 10^{-27} \mathrm{~kg}$ and $\boldsymbol{m}_{\mathbf{0}}=1,511593 \cdot 10^{-27} \mathrm{~kg}$.

Hence, we obtain the following formulas for the gravitational field:

The gravitational field of an "ordinary" body $\boldsymbol{M}$ :

$E=\frac{e_{1}}{r^{2}} \frac{M}{m}$ or $E=G \frac{M}{r^{2}}$, where $G=6,4242 \cdot 10^{-11}$

The gravitational field of a superdense body $\boldsymbol{M}_{\mathbf{0}}$ :

$E=\frac{e_{1}}{r^{2}} \frac{M_{0}}{m_{0}}$ or $E=G \frac{M_{0}}{r^{2}}$, where $G=\mathbf{7 , 0 5 7 2} \cdot \mathbf{1 0}^{-11}$

It should be noted that these Gs are of secondary importance in this study, because they do not reflect the physical essence of the gravity process.

The inclusion of concepts such as gravitational cells and gravitational strings in the concept of the gravitational field has led to other significant results. These results are the formula for the mass of a hydrogen atom and the formula for the mass of an electron.

When two superdense gravitational cells $\boldsymbol{m}_{\mathbf{0}}$ approach each other, the gravitational field between the cells reaches its maximum value equal to $\boldsymbol{E}=$ 
$\mathbf{1 , 0 2 6 7 8 9} \cdot \mathbf{1 0}^{-37}$. In this case, the gravitational string contracts and turns into a point gravitational quantum. In a situation of dense convergence of cells, the formula $E=\frac{e_{0}}{r^{2}} \frac{m_{0}}{m_{0}}$ ceases to operate and the Planck formula is valid:

$$
e_{0}=h \gamma
$$

where $\boldsymbol{e}_{\mathbf{0}}$ is the energy of the gravitational quantum of the black hole, $\boldsymbol{e}_{\mathbf{0}}=$ $1,026789 \cdot 10^{-37} \mathrm{~J}$.

$\boldsymbol{h}$ is Planck's constant, $\mathbf{6 , 6 2 6 0 7} \cdot \mathbf{1 0}^{-34} \mathrm{~J} \cdot \mathrm{s}$. $\boldsymbol{\gamma}$ - frequency, where $=\frac{\boldsymbol{e}_{\mathbf{0}}}{\boldsymbol{h}}=\mathbf{1 , 5 4 9 6 2 0} \cdot \mathbf{1 0}^{-\mathbf{4}} \mathrm{s}^{-1}(\boldsymbol{\gamma}$ is constant and does not depend on the SI or CGSE measurement system).

The $\boldsymbol{\gamma}$ frequency is related to the proportion between the energy-mass of negative and positive charges in the gravitational quantum:

$$
\gamma=\frac{1}{4} \frac{2 e_{-e}}{2 e_{+p}}=\frac{1}{4} \frac{e_{-e}}{e_{+p}}
$$

$\boldsymbol{e}_{-\boldsymbol{e}}$ is the value of the energy of the negative charge inside the cell, J.

$\boldsymbol{e}_{+\boldsymbol{p}}$ - the value of the energy of the positive charge inside the cell, J.

Hence, for $\boldsymbol{\gamma}=\frac{\boldsymbol{e}_{\mathbf{0}}}{\boldsymbol{h}}$, we obtain the formula:

$$
\frac{e_{-e}}{e_{+p}}=\frac{4 e_{0}}{h}
$$

The definition of "energy-mass of negative and positive charge" was introduced because electrons and protons are not independent particles inside gravitational cells and gravitational quanta. The fraction $\mathbf{1 / 4}$ is explained by the fact that four charges are involved in the formation of a gravitational quantum, while only one charge in the form of an electron participates in the formation of an 
electromagnetic quantum. (The correctness of formulas (1-11) and (1-12) will be confirmed below by the coincidence of calculations by these formulas with experimental data

Based on f. (1-11), (1-12) and taking into account that the total energy of the gravitational cell is $\boldsymbol{m}_{\mathbf{0}} \boldsymbol{c}^{2}=\boldsymbol{e}_{+\boldsymbol{p}}+\boldsymbol{e}_{-\boldsymbol{e}}$, we obtain the formula for the energy of a negative charge inside a superdense cell:

$$
e_{-e}=\frac{4 e_{0} m_{0} c^{2}}{h+4 e_{0}}
$$

Taking into account that $\boldsymbol{m}_{\mathbf{0}}=\frac{\sqrt{\mathbf{8}} \boldsymbol{q}}{\boldsymbol{c}}$ and $\boldsymbol{e}_{\mathbf{0}}=\mathbf{4} \boldsymbol{q}^{2}$, we obtain the value $\boldsymbol{e}_{-\boldsymbol{e}}$ through the formula with three fundamental constants:

$$
e_{-e}=\frac{32 \sqrt{2} q^{3} c}{\left(h+16 q^{2}\right)}=8,415740 \cdot 10^{-14} \mathrm{~kg}
$$

As you can see, $\boldsymbol{e}_{-\boldsymbol{e}}$ almost coincided with the energy of a free electron, where $\boldsymbol{e}=\mathbf{8 , 1 8 7 1 1 0} \cdot \mathbf{1 0}^{-\mathbf{1 4}} \mathrm{J}$. The discrepancy is $\mathbf{2 , 7 \%}$. (It should be noted that there should not be a complete coincidence here, because the negative charge inside the gravitational cell and the free electron are different physical quantities).

The energy value of the positive charge in the cell will be: $\boldsymbol{e}_{+\boldsymbol{p}}=\boldsymbol{m}_{\mathbf{0}} \boldsymbol{c}^{\mathbf{2}}-$ $e_{-e}=1,511593 \cdot 10^{-27} \cdot c^{2}-8,415740 \cdot 10^{-14}=13,577104 \cdot 10^{-11} \mathrm{~J}$. The ratio between the energies of elementary charges in a superdense cell is: $\frac{\boldsymbol{e}_{-\boldsymbol{e}}}{\boldsymbol{e}_{+p}}=$ $\frac{4 e_{0}}{h}=6,19848 \cdot 10^{-4}$

The body of a black hole consists of a huge set of such closely spaced superdense cells. Now let's consider the process of formation of hydrogen atoms from gravitational cells. For this, external energy must enter each cell. As a result 
of energy entering the cell, the positive charge in the cell $\boldsymbol{e}_{+\boldsymbol{p}}=\mathbf{1 3}, \mathbf{5 7 7} 104$.

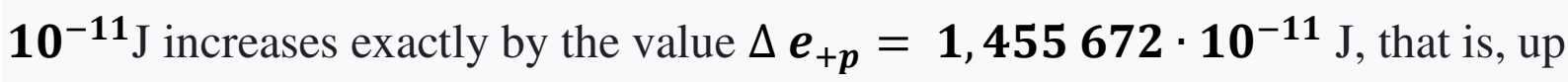

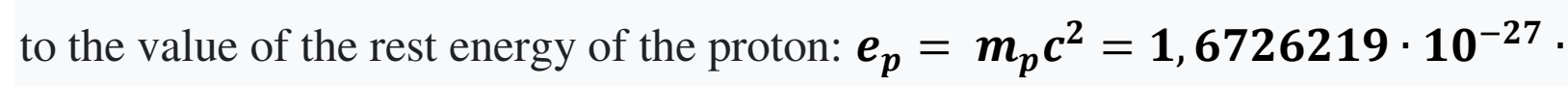
$c^{2}=15,032776 \cdot \mathbf{1 0}^{-11} \mathrm{~J}$. (More than this value, the positive charge in the cell cannot increase). Thus, the total energy of the gravitational cell increases by $\Delta \boldsymbol{e}_{+\boldsymbol{p}}$ and becomes equal to:

$$
e=e_{-e}+e_{p}=8,415740 \cdot 10^{-14}+15,032776 \cdot 10^{-11}=
$$

\section{$15,041191 \cdot 10^{-11} \mathrm{~J}$}

As you can see, the calculated value $\boldsymbol{e}=\mathbf{1 5}, \mathbf{0 4 1 1 9 1} \cdot \mathbf{1 0}^{-\mathbf{1 1}} \mathrm{J}$ almost absolutely coincided with the experimental value of the energy of the hydrogen atom, where $\boldsymbol{e}_{\boldsymbol{h}}=\mathbf{1 , 6 7 3 5 5 7 5} \cdot \mathbf{1 0}^{-27} \cdot \boldsymbol{c}^{2}=\mathbf{1 5 , 0 4 1 1 8 5} \cdot \mathbf{1 0}^{-11} \mathrm{~J}$. The ultramicroscopic discrepancy of $\mathbf{6} \cdot \mathbf{1 0}^{-\mathbf{1 7}} \mathrm{J}$ can be safely attributed to the permissible error of calculations. But at the same time, this very small discrepancy may be a consequence of the release of neutrino particles during the formation of a hydrogen atom (here it is necessary to take into account that $\mathbf{1 5 , 0 4 1 1 9 1}$. $\mathbf{1 0}^{-11} \mathrm{~J}>\mathbf{1 5 , 0 4 1 1 8 5} \cdot \mathbf{1 0}^{-11} \mathrm{~J}$, and not vice versa). Therefore, taking into account this probability, we write the result obtained by the following formula:

$$
e_{h}=\frac{32 \sqrt{2} q^{3} c}{\left(h+16 q^{2}\right)}+e_{p}-\sum e_{v}
$$

where $\boldsymbol{e}_{\boldsymbol{h}}$ is the energy of a hydrogen atom, $\mathbf{1 5 , 0 4 1 1 8 5} \cdot \mathbf{1 0}^{-11} \mathrm{~J}$.

$\boldsymbol{e}_{\boldsymbol{p}}$ is the rest energy of a proton, $\mathbf{1 5}, \mathbf{0 3 2 7 7 6} \cdot \mathbf{1 0}^{-\mathbf{1 1}} \mathrm{J}$.

$\sum \boldsymbol{e}_{v}$ - total neutrino energy, $\sum \boldsymbol{e}_{v}=\mathbf{6} \cdot \mathbf{1 0}^{-\mathbf{1 7}} \mathrm{J}$. 
$\boldsymbol{q}, \boldsymbol{h}, \boldsymbol{c}$ - fundamental physical constants.

(It should be noted that after the formation of a hydrogen atom from a

superdense cell, the ratio between the charge energies $\left(\frac{\boldsymbol{e}_{-e}}{\boldsymbol{e}_{+p}}=\mathbf{6}, \mathbf{1 9 8 4 8} \cdot \mathbf{1 0}^{-\mathbf{4}}\right)$ changes and becomes equal: $\frac{e_{-e}}{e_{+p}}=\frac{4 e}{h}=\frac{4 \cdot 1,108293 \cdot 10^{-37}}{h}=6,6905 \cdot 10^{-4}$ )

Taking into account that $\boldsymbol{E}=\boldsymbol{m} \boldsymbol{c}^{\mathbf{2}}$, we get the formula for the mass of a hydrogen atom:

$$
m_{h}=\frac{32 \sqrt{2} q^{3}}{c\left(h+16 q^{2}\right)}+m_{p}-\sum m_{v}
$$

where $\boldsymbol{m}_{\boldsymbol{h}}$ is the mass of a hydrogen atom, $\mathbf{1 , 6 7 3 5 5 7 5} \cdot \mathbf{1 0}^{-\mathbf{2 7}} \mathrm{kg}$.

$\boldsymbol{m}_{\boldsymbol{p}}$ is the mass of the proton, $\mathbf{1}, \mathbf{6 7 2 6 2 1 9} \cdot \mathbf{1 0}^{-\mathbf{2 7}} \mathrm{kg}$.

$\sum \boldsymbol{m}_{v}$ is the total mass of the neutrino, $\sum \boldsymbol{m}_{v}=\mathbf{7} \cdot \mathbf{1 0}^{-\mathbf{3 4}} \mathrm{kg}$.

$\boldsymbol{q}, \boldsymbol{h}, \boldsymbol{c}$ - fundamental physical constants.

Considering that the value $\sum \boldsymbol{m}_{v}$ is negligible in relation to the mass of a hydrogen atom $(0,00004 \%)$, formula (1-16) can be written as:

$$
m_{h}=\frac{32 \sqrt{2} q^{3}}{c\left(h+16 q^{2}\right)}+m_{p}(1-18)
$$

Thus, the calculated mass of the hydrogen atom almost completely coincided with the experimental mass of the hydrogen atom. This result directly confirms the correctness of formulas (1-11), (1-12), as well as the value obtained using the formula for the Schwarzschild radius $\boldsymbol{m}_{\mathbf{0}}=\frac{\sqrt{\mathbf{8 q}}}{\boldsymbol{c}}=\mathbf{1}, \mathbf{5 1 1 5 9 3} \cdot \mathbf{1 0}^{-\mathbf{2 7}}$ $\mathrm{kg}$. 
(For a better perception of information, some formulas do not specifically set conversion factors for units of measurement, which are equal to 1).

\section{Results.}

The main results of this study should include the introduction of such important components as gravitational cells and gravitational strings into the concept of the gravitational field. This circumstance made it possible to obtain new physical formulas and indicators. This should include the formula for the gravitational constant, the formula for the gravitational quantum, the formula for the proportion between the mass-energy of the electron and the proton, the formula for the mass of the hydrogen atom, the mass of the gravitational cell, the value of the gravitational quantum, etc. All the results obtained using the new formulas are fully correlated with the experimental data. Some of the indicators obtained by the formulas coincide with the experimental indicators with an accuracy of $10^{-7}$ (the mass of a hydrogen atom). At the same time (which is especially important) all new formulas are built on a single theoretical basis (gravitational cells and gravitational strings) and therefore have the same physical constants (speed of light, Planck's constant, elementary charge). This circumstance is an additional confirmation of the correctness of the inclusion of new physical concepts in the gravitational interaction. 


\section{Conclusion.}

In this work, for the first time, it was possible to build into the gravitational field such concepts as gravitational cells and gravitational strings. This made it possible to obtain such scientific results as the formula for the gravitational constant, the formula for the proportion between the mass-energy of the electron and the proton, the formula for the hydrogen atom, etc. These formulas were fully confirmed by experimental data. In this study, Planck's formula was successfully embedded in the gravitational field and this led to practical results. In this work, the concept of a gravitational quantum is introduced and its value is precisely determined. Also, a new physical constant was obtained - the mass of the gravitational cell of a black hole.

Further research in this direction will be continued. 


\section{Declarations}

1. Availability of data and materials.

All data obtained and analyzed in the course of this study is included in this article.

2. Competing interests. Not applicable (there are no competing interests).

3. Funding. Not applicable.

4. Authors' contributions. Not applicable.

5. Acknowledgements. Not applicable. 